\title{
Reduction of Coil Mass Artifacts in High-Resolution Flat Detector Conebeam CT of Cerebral Stent-Assisted Coiling
}

\author{
I.M.J. van der Bom, S.Y. Hou, A.S. Puri, G. Spilberg, D. Ruijters, P. van de Haar, B. Carelsen, S. Vedantham, M.J. Gounis, and A.K. Wakhloo
}

\begin{abstract}
BACKGROUND AND PURPOSE: Developments in flat panel angiographic C-arm systems have enabled visualization of both the neurovascular stents and host arteries in great detail, providing complementary spatial information in addition to conventional DSA. However, the visibility of these structures may be impeded by artifacts generated by adjacent radio-attenuating objects. We report on the use of a metal artifact reduction algorithm for high-resolution contrast-enhanced conebeam CT for follow-up imaging of stent-assisted coil embolization.
\end{abstract}

MATERIALS AND METHODS: Contrast-enhanced conebeam CT data were acquired in 25 patients who underwent stent-assisted coiling. Reconstructions were generated with and without metal artifact reduction and were reviewed by 3 experienced neuroradiologists by use of a 3-point scale.

RESULTS: With metal artifact reduction, the observers agreed that the visibility had improved by at least 1 point on the scoring scale in $>40 \%$ of the cases $(\kappa=0.6)$ and that the streak artifact was not obscuring surrounding structures in $64 \%$ of all cases $(\kappa=0.6)$. Metal artifact reduction improved the image quality, which allowed for visibility sufficient for evaluation in $65 \%$ of the cases, and was preferred over no metal artifact reduction in $92 \%(\kappa=0.9)$. Significantly higher scores were given with metal artifact reduction $(P<.0001)$.

CONCLUSIONS: Although metal artifact reduction is not capable of fully removing artifacts caused by implants with high x-ray absorption, we have shown that the image quality of contrast-enhanced conebeam CT data are improved drastically. The impact of the artifacts on the visibility varied between cases, and yet the overall visibility of the contrast-enhanced conebeam CT with metal artifact reduction improved in most the cases.

ABBREVIATIONS: $M A R=$ metal artifact reduction; $\mathrm{CBCT}=$ conebeam $\mathrm{CT}$; VasoCT $=$ high-resolution contrast-enhanced $\mathrm{CBCT}$

$\mathbf{E}$ ver since its introduction, the stent-assisted coil embolization technique has broadened the field for endovascular treatment of intracranial aneurysms to wide-neck aneurysms. ${ }^{1-8}$ The use of neurovascular stents that serve as a scaffold allows for higher coil packing densities with a relatively low chance of coils herniating into parent arteries. ${ }^{9}$ Currently, its application is not limited only to giant and fusiform aneurysms but it is also being used for smaller berrylike aneurysms. ${ }^{10}$

Received October 11, 2012; accepted after revision January 28, 2013.

From the Department of Radiology (I.M.J.v.d.B., S.V.H., A.P., G.S., S.V., M.J.G., A.K.W.), New England Center for Stroke Research, University of Massachusetts Medical School, Worcester, Massachusetts; and Interventional X-Ray (D.R., P.v.d.H., B.C.), Philips Healthcare, Best, The Netherlands.

This article received funding from (RO1 CA128906).

Please address correspondence to I.M.J. van der Bom, PhD, University of Massachusetts, Department of Radiology, New England Center for Stroke Research, 55 Lake Ave North, SA-107F, Worcester, MA 01655; e-mail: martijn.vanderbom@umassmed.edu

- Indicates open access to non-subscribers at www.ajnr.org

http://dx.doi.org/10.3174/ajnr.A3561
Because of the potential risk of aneurysm regrowth and of in-stent stenosis with the use of neurovascular stents, careful patient monitoring after endovascular treatment is essential. Patient follow-up is conventionally performed by catheter-based DSA because it provides a high spatial and temporal resolution. However, a disadvantage of this technique is that it only provides $2 \mathrm{D}$ information of the vascular anatomy, and the relationship of the vascular anatomy to the stent and coil mass may not be fully appreciated.

The latest generation of angiographic C-arm systems equipped with flat panel technology not only provide conventional 2D fluoroscopy but enable in situ 3D conebeam CT (CBCT) that can be can used for peri-interventional evaluation. ${ }^{11}$ Recently, the development and application of high-resolution contrast-enhanced conebeam CT (VasoCT; Philips, Best, the Netherlands) with the use of an angiographic flat-panel C-arm system has been reported. ${ }^{12,13}$ This technique enables detailed 3D visualization of neurovascular stents and host arteries that allows 

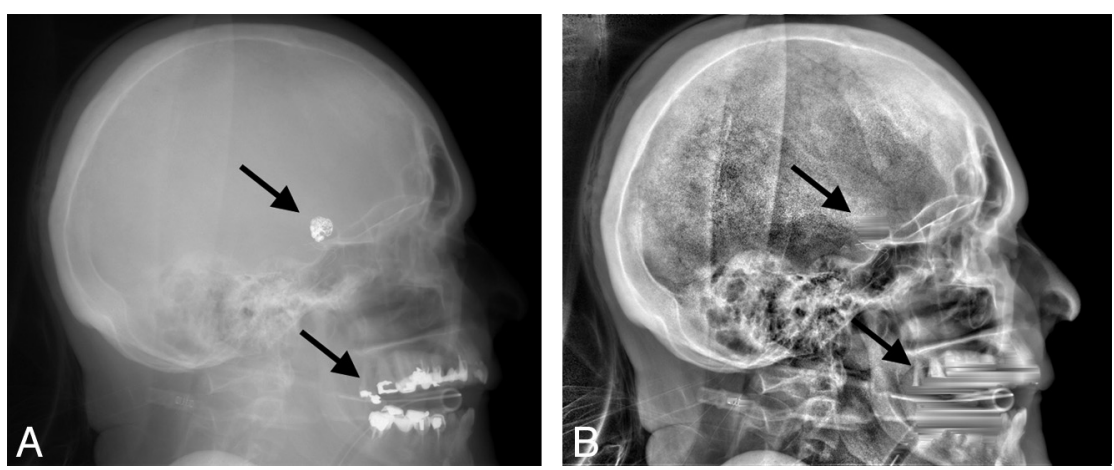

FIG 1. Example x-ray image acquired by the flat panel angiographic C-arm system before (left) and after (right) removal and replacement of the high-absorption areas caused by coils and dental fillings (arrows).
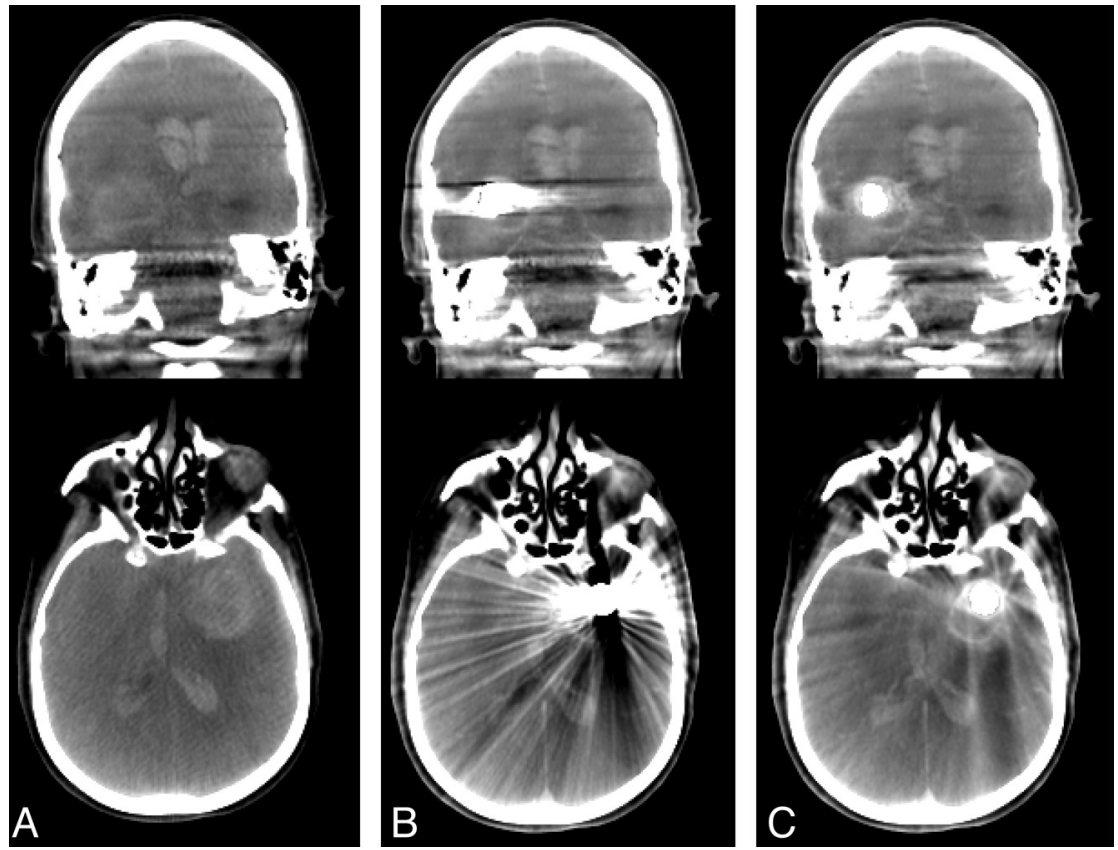

FIG 2. Coronal (top row) and axial sections (bottom row) of conebeam CT data of a patient acquired before coil embolization (column A), after coil embolization without MAR (column B), and after coil embolization with MAR (column C).

for a more complete determination of stent-wall apposition and in-stent stenosis. However, in cases of stent-assisted coiling, the high x-ray absorption of the coil mass generates streak artifacts that obscure surrounding structures and therefore severely limit the diagnostic quality of the acquisition.

In the 1980s, Glover and Pelc ${ }^{14}$ and Kalender et al $^{15}$ suggested manipulating raw data before reconstruction to reduce the effect of metallic implants in CT. Since these first reports, a diversity of similar algorithms have been proposed that address the occurrence of metal artifacts in multidetector CT data. Various methods that aim to replace sinogram data have been investigated, of which most explore different methods of segmentation and interpolation. ${ }^{15-21}$ Veldkamp et $\mathrm{al}^{18}$ have shown, however, that replacement of missing sinogram data with more advanced routines than linear interpolation has a minor impact on the image quality. Other suggested methods to reduce metal artifacts include dual energy, ${ }^{22}$ iterative reconstruction, ${ }^{23-25}$ manipulation of recon- structed CT data, ${ }^{26,27}$ and combinations of methods. ${ }^{28}$ Thus far, these methods have not found their way into clinical routine, which is mainly caused by their computational complexity. Prell et $\mathrm{al}^{29,30}$ have elaborated on the method proposed by Kalender et $\mathrm{al}^{15}$ for CBCT, which replaces underexposed pixels in the raw projection images rather than sinograms. Their results showed an overall improvement of visibility of neurovascular implants and surrounding brain tissue.

Our research objective was to determine whether application of a multipass reconstruction algorithm that reduces the artifacts caused by implants with high $\mathrm{x}$-ray absorption improves the visibility of VasoCT data. The effects of this technique on the diagnostic image quality of VasoCT data acquired after stent-assisted coil embolization were evaluated by an observer study. In the following section, a brief description of the multipass reconstruction algorithm used for metal artifact reduction (MAR) is given.

\section{MATERIALS AND METHODS}

The MAR algorithm used in our study is based on the algorithm proposed by Prell et al. ${ }^{29,30}$ With this method, a reconstruction is generated during the first pass by use of a regular filtered back-projection algorithm. ${ }^{31}$ Regions within the primary reconstruction that display relatively high $\mathrm{x}$-ray absorption are automatically isolated from the volumetric data by use of a predefined threshold value of 4000 HU. Subsequently, the volumetric regions of high absorption are mapped onto the original $\mathrm{x}$-ray images by forward projection, ${ }^{32}$ which is achieved by accurate geometric calibration of the C-arm system. ${ }^{33}$ The high-absorption regions within the original $\mathrm{x}$-ray images are replaced by gray-values linearly interpolated from the surrounding scan lines (Fig 1). Finally, a new reconstruction is formed by filtered back-projection in the second pass, with the use of the adjusted $\mathrm{x}$-ray images (Fig 2). Reconstruction of a CBCT volume with matrix $256^{3}$ (voxel size, $0.2^{3}$ $\mathrm{mm}^{3}$ ) with and without MAR takes approximately 72 and 138 seconds, respectively, on a Dual Core Xeon processor (Intel, Santa Clara, California).

Thirty patients $(66.7 \%$ women; mean age, 57.2 years; age range, 36-81 years) who underwent stent-assisted coil embolization were retrospectively included in this study. Mean \pm standard deviation aneurysm size and packing density were $5.5 \pm 4.6 \mathrm{~mm}$ (range, $1.8-27 \mathrm{~mm}$ ) and $46.3 \pm 39.4 \%$, respectively. In $90 \%$ of the cases, the aneurysm was located in the anterior circulation. High- 


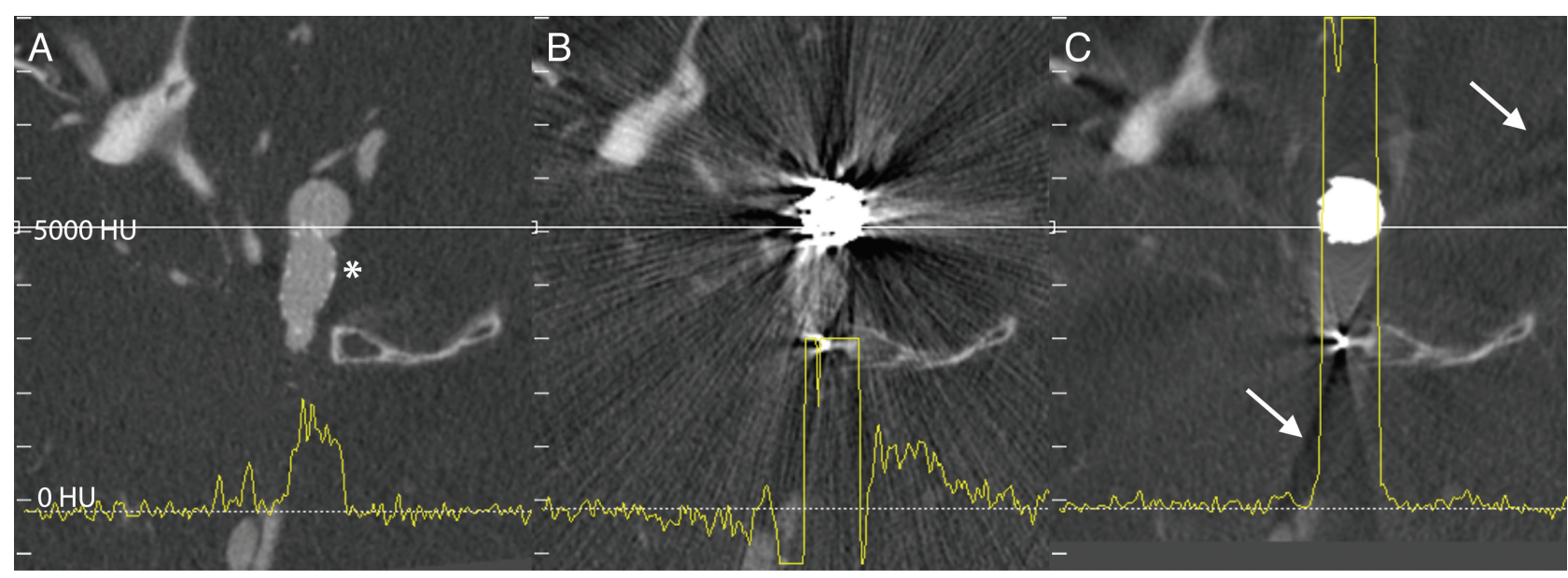

FIG 3. Corresponding axial sections of VasoCT data acquired after stent placement ( $A$, stent indicated by asterisk) and after coil embolization $(B$ and C). Streak artifacts generated by the coil mass visible in VasoCT data without MAR (B) are severely reduced with MAR (C). Because of the replacement of absent data in the raw projections, subtle new artifacts appear in VasoCT with MAR (C, arrows). Intensity profiles (yellow lines) were generated for all 3 images by use of the same physical coordinates (white lines). The intensity scale of the profile analysis is given on the left-hand side of the figure. Profile plots show that severe fluctuations outside the coil mass are reduced by MAR and the resulting profile in $C$ is similar to the profile in $A$.

resolution contrast-enhanced CBCT data were obtained immediately after stent-assisted coil embolization or at 6-month followup, as is regularly performed at our institution. Image data were acquired with a flat panel angiographic C-arm system (Allura Xper FD20, Philips) by use of a reduced detector size of $22 \mathrm{~cm}$ to obtain high-resolution nonbinned images. Iodinated contrast (Iopamidol 51\%, $510 \mathrm{mg} / \mathrm{mL}$, Isovue; Bracco Diagnostics, Princeton, New Jersey) diluted to $20 \%$ was injected from the internal carotid artery with $3 \mathrm{~mL} / \mathrm{s}$, or from the vertebral artery with 2 $\mathrm{mL} / \mathrm{s}$ for a total of 23 seconds by use of a $5 \mathrm{~F}$ or $6 \mathrm{~F}$ catheter and an imaging delay of 2 seconds. VasoCT volumes were generated from $621 \mathrm{x}$-ray images $(80 \mathrm{kv}, 260 \mathrm{~mA})$ with a matrix size of $1016^{2}$ (pixel size: $0.15^{2} \mathrm{~mm}^{2}$ ) obtained during the rotational sweep of the $\mathrm{x}$-ray source of approximately $200^{\circ}$ in 20 seconds and by use of a filtered back-projection reconstruction algorithm. For all included patients, VasoCT data were generated both with and without usage of the metal artifact reduction algorithm and were reviewed in a blinded fashion by 3 experienced neuroradiologists on a dedicated workstation and a medical grade monitor. The diagnostic quality of both reconstructions were rated by means of a 3 -point scale ( 1 = insufficient for evaluation, $2=$ sufficient for evaluation, 3 = excellent) questionnaire addressing the: 1) visibility of the stent directly adjacent to the coil mass, 2) the visibility of the host artery directly adjacent to the coil mass, and 3) the visibility of the relationship between the aneurysm, host artery, stent, and coil mass. In addition, the observers were asked whether streak artifacts were obscuring the vessel beyond the actual coil mass (yes/no) and which of the 2 reconstructions offered overall better visibility. During review, observers were allowed to adjust window-level settings and section thickness of the image data as well as zoom, pan, and rotate to optimize viewing. A general consensus regarding the 3-point scale was established with the use of 5 cases that were excluded from the actual observer study. The overall agreements were calculated by means of raw statistics, and the reliability of agreement was analyzed by the Fleiss $\kappa$ method. ${ }^{34}$ Significance of the results was evaluated by means of Fisher exact test for the categoric and yes/no questions. A 2-tailed significance level of $P<.05$ was considered significant. Intraobserver and interobserver analyses were performed by means of a Wilcoxon matched-pairs signed-rank test. Statistical analyses were performed by means of Prism Five (GraphPad, San Diego, California).

\section{RESULTS}

The effect of MAR on VasoCT data of a patient who underwent stent-assisted coil embolization is demonstrated in example data given in Fig 3. Corresponding axial sections of VasoCT data acquired immediately after stent placement (Fig 3A, stented segment is indicated by the asterisk), after coil embolization without MAR (Fig 3B), and after coil embolization with MAR (Fig 3C) are shown. Comparison of Figs $3 B$ and $3 C$ reveals the evident reduction of streak artifacts caused by the coil mass. Although the streak artifacts caused by the coil mass were mostly eliminated, new, more subtle streak artifacts appear in VasoCT data with MAR (indicated by the arrows in Fig 3C), which is caused by interpolation of missing data in the raw projection images. The effect of subtle streak artifacts caused by the MAR algorithm is demonstrated more clearly in Fig 4. Profile plots in Fig 3 (yellow line) are generated of the same physical coordinates (white line) of VasoCT data. The intensity scales are indicated on the left of each image and range from -1200 to $9000 \mathrm{HU}$. It should be noted that for reconstructions without MAR and with MAR, intensities are automatically truncated at $3000 \mathrm{HU}$ and $9000 \mathrm{HU}$, respectively. Low-frequency fluctuations adjacent to the coil mass present in the profile plot in Fig $3 B$ are removed by MAR. As a result, the profiles in Figs $3 A$ and $3 C$ have a similar noise pattern outside the coil mass.

The results of the observer study are summarized in the Table. The average overall agreement of the observer study was $78 \%$. In more than half of the cases without MAR, all observers agreed that the visibility of the stent, the host artery, and relationship between aneurysm, host artery, stent, and coil mass was insufficient for 
evaluation (score of $1, \kappa=0.7$ ). In addition, the observers agreed in $56 \%$ of all cases that the artifact was obscuring the host artery beyond the actual coil mass when MAR was not used.

With MAR, the number of cases in which the observers agreed on giving a score of 1 (insufficient for evaluation) was reduced by $>50 \%$ for the visibility of the stent and host artery. The visibility of the relationship between the stent, host artery, the aneurysm, and the coil mass was improved by at least 1 point on the scoring system in $40 \%$ of the cases $(\kappa=0.6)$, and in $36 \%$ of the cases, the observers agreed that the visibility was sufficient for evaluation
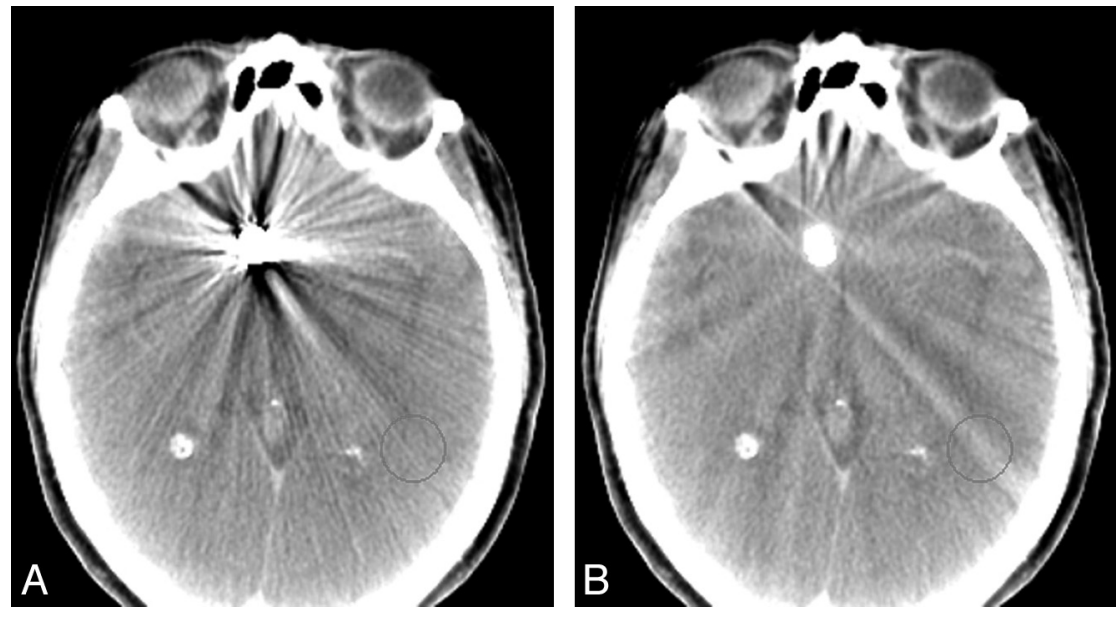

FIG 4. Conebeam CT data without (A) and with (B) MAR demonstrating the reduction of coil mass artifacts and the appearance of subtle streak caused by the algorithm, which is specifically well visualized in the indicated circular region of interest. (score $\geq 2$ ). In $64 \%$ of the cases, the observers agreed that the streak artifact was not obscuring the host artery and on average the overall visibility of the VasoCT data were sufficient for evaluation (score $\geq 2$ ) in $65 \%$ of the cases with MAR. The observers concluded with high overall agreement $(92 \%, \kappa=0.9)$ that the overall visibility was improved when MAR was used. VasoCT data with MAR were rated with significantly higher scores $(P<.05)$ than without MAR for all categories. A Wilcoxon matched-pairs signed-rank test showed that for all categories, the pooled and unpooled scores given by the observers to VasoCT data without MAR were significantly improved when the MAR algorithm was used $(P<$ .0001). Intraobserver analysis performed by a single observer revealed no significant difference in scoring $(P>.5)$.

\section{Illustrative Cases}

Case 1. A 51-year-old woman had a history of subarachnoid hemorrhage secondary to a ruptured right middle cerebral artery aneurysm that was treated by surgical clipping. On the diagnostic cerebral angiogram obtained for evaluation of the surgical clipping procedure, an incidental unruptured wide-neck left posterior communicating artery aneurysm was observed. This posterior communicating artery aneurysm was endovascularly treated by stent-assisted coil

Results of the observer study rating the visibility without and with MAR

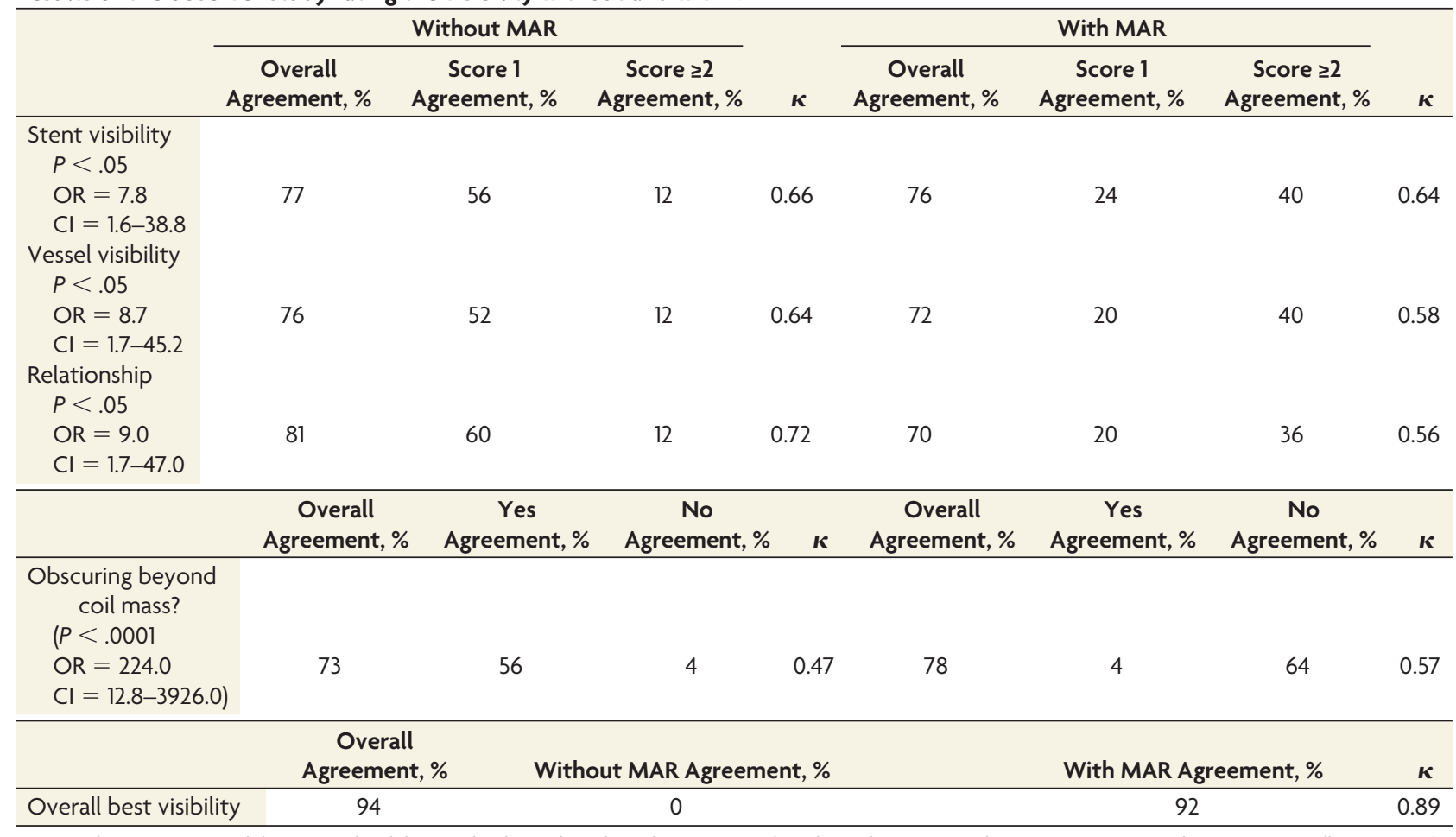

Note:-The rows "stent visibility," "vessel visibility," and "relationship" show the summarized results to the 3-point scale questions. Given are the percent overall agreement (ie, the number of cases that all reviewers agreed in total, calculated using the Fleiss method), the percent agreement for a score 1 (ie, the number of cases all reviewers agreed on giving a score of 1), and the percent agreement for a score $\geq 2$ (ie, the number of cases all reviewers agreed on giving a score of 2 or 3 ). Similarly, agreements for the binary questions are indicated. For each observer question, the $\kappa$ values, $P$ value, odds ratio (OR), and $95 \%$ confidence intervals (CI) are given when applicable. OR represents the improvement of classification of 1 to $\geq 2$ with MAR. 

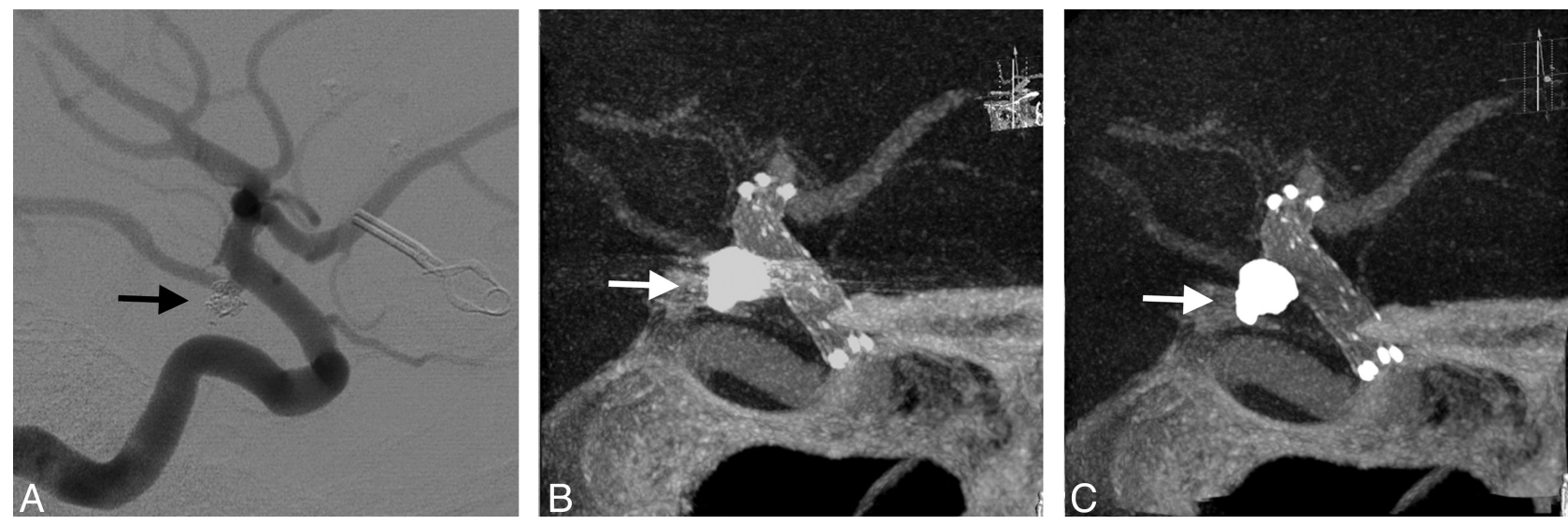

FIG 5. Illustrative case 1. DSA at 6-month follow-up shows no recanalization of the embolized posterior communicating artery aneurysm (A) Streak artifacts caused by the coil mass (arrows) in maximum intensity projection of VasoCT data without MAR (B) partially obscures visualization of stent and host artery. After MAR (C), streak artifacts in VasoCT data were removed, revealing the stent and host artery.
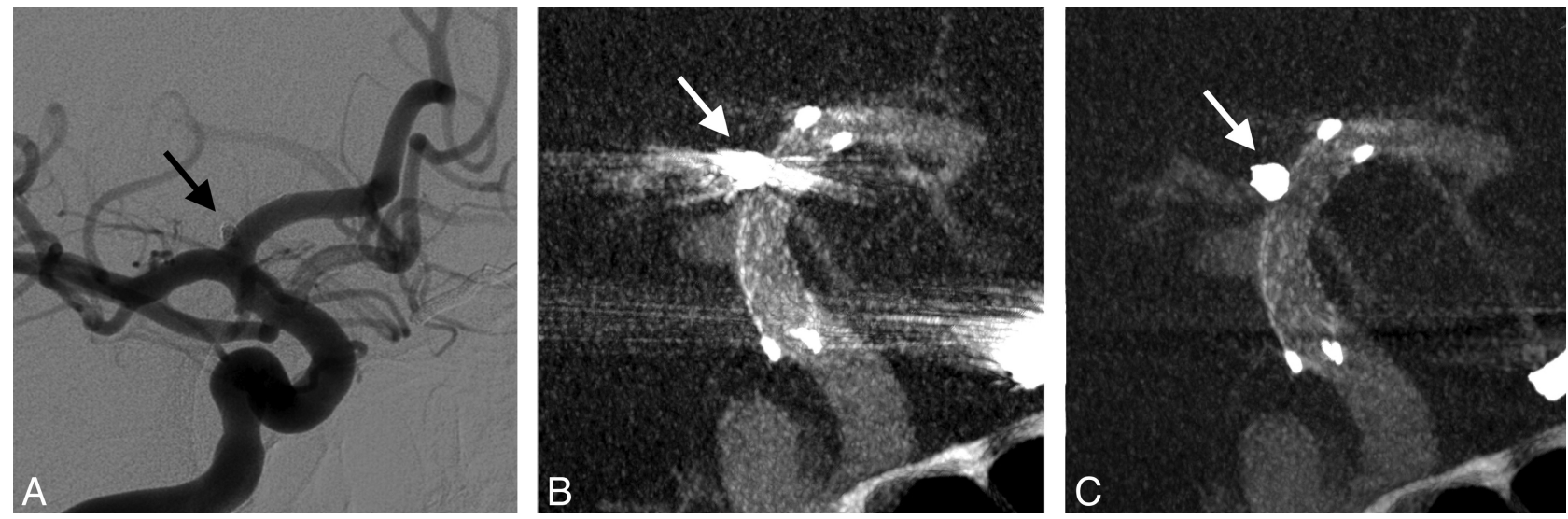

FIG 6. Illustrative case 2. Immediate DSA (A) maximum intensity projection of VasoCT data without MAR (B) and with MAR (C) of stent-assisted coil embolized aneurysm at the right A1 segment. Visibility is significantly affected by streak artifacts caused by the coil mass (arrows) and contralateral clip in VasoCT without MAR. With MAR, stent apposition to the vascular wall is fully appreciated.

embolization technique. The illustrated case (Fig 5) shown was the 6-month follow-up VasoCT examination of her left posterior communicating artery aneurysm. This case received the following median scores without MAR versus with MAR, respectively: stent visibility: 2 versus 2; vessel visibility: 2 versus 3 ; and relationship: 2 versus 3 .

Case 2. A 40-year-old man with a ruptured left ophthalmic artery aneurysm underwent surgical clip ligation. The diagnostic cerebral angiogram examination for postsurgical evaluation revealed an incidental unruptured posteriorly projecting small aneurysm at the Al segment of the right anterior communicating artery. This second aneurysm was endovascularly treated by stent-assisted coil embolization. Fig 6 shows the immediate posttreatment DSA and VasoCT examinations. This case received the following median scores without MAR versus with MAR, respectively: stent visibility: 2 versus 3; vessel visibility: 2 versus 3; and relationship: 2 versus 3 .

Case 3. A 61-year-old woman with a family history of brain aneurysms was brought in to our hospital for a diagnostic work-up. MRA examination of the head showed a left internal carotid artery terminus aneurysm that was treated by stent-assisted coiling. The illustrated case (Fig 7) shown was the immediate posttreatment DSA and VasoCT examination. This case received the following median scores without MAR versus with MAR, respectively: stent visibility: 2 versus 2; vessel visibility: 2 versus 3; and relationship: 2 versus 3 .

Case 4. A 64-year-old woman with a family history of ruptured intracranial aneurysms underwent a diagnostic work-up for her chronic dizziness. MRA revealed bilateral unruptured (middle cerebral artery) brain aneurysms. Both aneurysms were treated by coil embolization; however, the right middle cerebral artery bifurcation aneurysm was treated by stent-assisted technique. In Fig 8, DSA and VasoCT acquired immediately after embolization are shown. This case received the following median scores without MAR versus with MAR, respectively: stent visibility: 1 versus 2; vessel visibility: 2 versus 2; and relationship: 2 versus 2 .

\section{DISCUSSION}

Stent-assisted coil embolization is very effective for treating fusiform and wide-neck aneurysms. This technique enables improved packing density with a relative low risk of coils herniating into the parent artery, which may also be beneficial for small to medium aneurysms. ${ }^{35}$ In addition, a fully deployed stent may function as a scaffold for endothelial growth. ${ }^{7}$ 

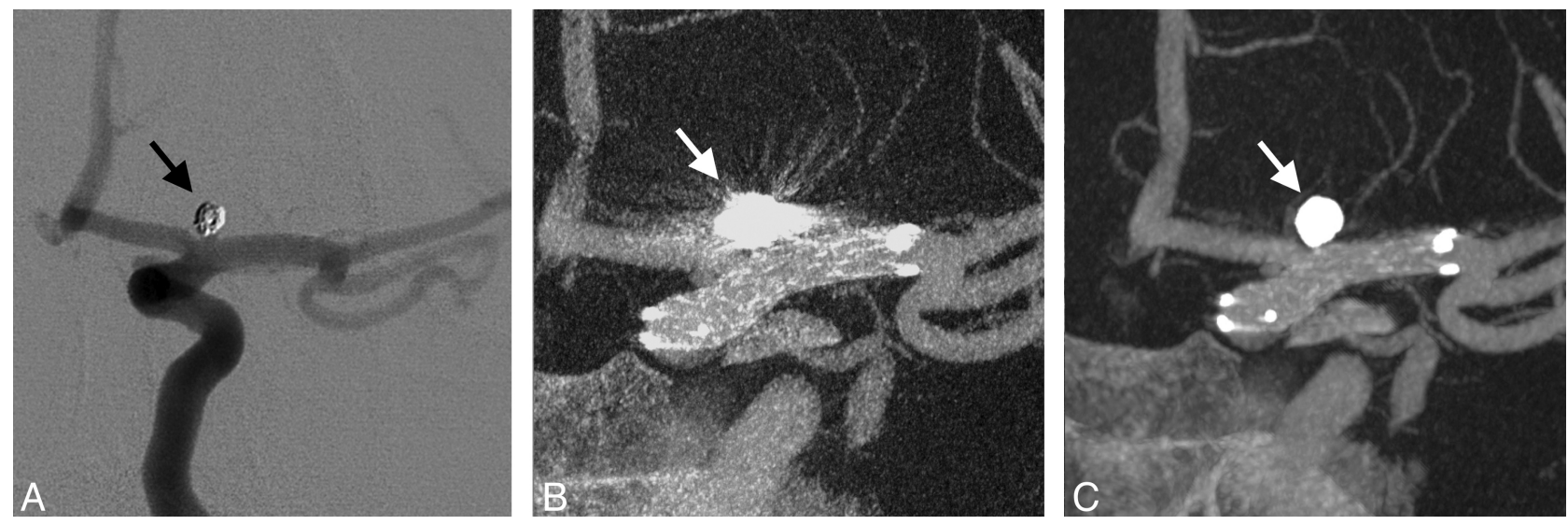

FIG 7. Illustrative case 3. DSA (A), maximum intensity projections of VasoCT without (B), and with MAR (C) acquired immediately after stent-assisted coil (arrows) embolization procedure. Streak artifacts partially obscuring the host artery and side branches are removed by the MAR method.
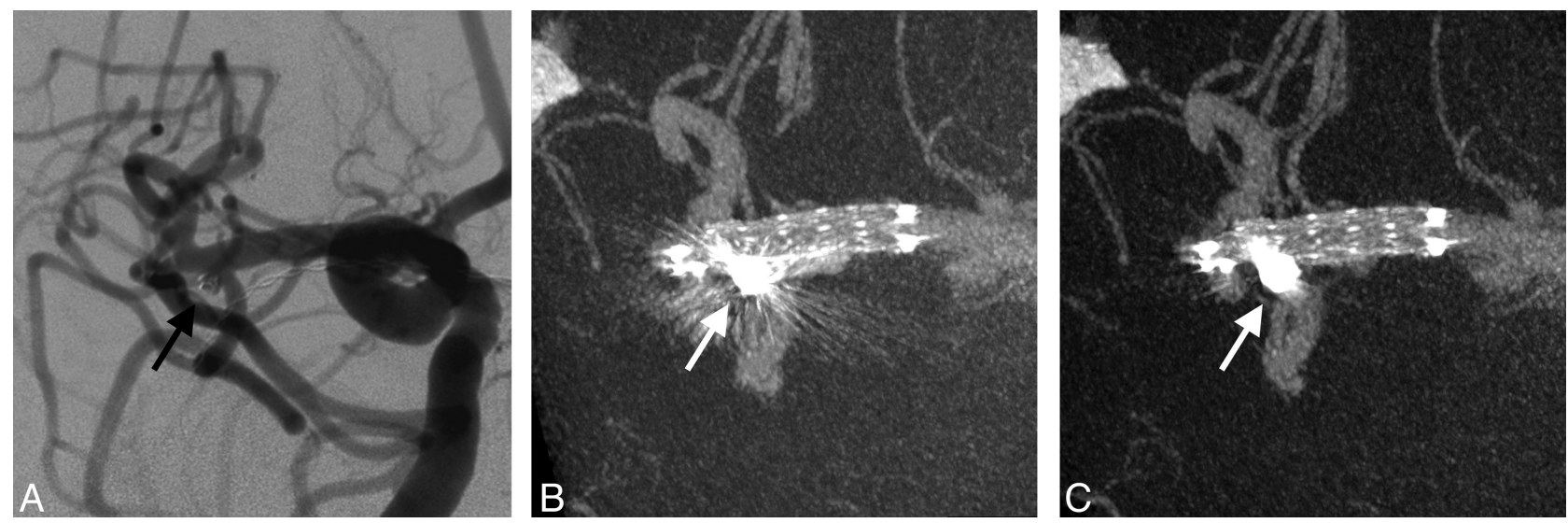

FIG 8. Illustrative case 4. Immediate DSA (A), VasoCT without MAR (B), and VasoCT with MAR (C) after stent-assisted coiling of an unruptured middle cerebral artery aneurysm. Although streak artifacts caused by the coil mass (arrows) are significantly reduced, a small amount of streak remains after application of MAR.

The standard technique for follow-up imaging after (stentassisted) coil embolization of intracranial aneurysms is DSA, which only provides a $2 \mathrm{D}$ projection of the vascular anatomy and implants. Diagnostic interpretation is therefore determined by the projection angle of the $\mathrm{x}$-ray source and may not fully disclose adjacent vascular anatomy and potential clot formation, stentwall apposition, stent herniation, recanalization, intimal tissue growth, or hyperplasia. In situ-acquired high-resolution contrast-enhanced CBCT (VasoCT) allows visualization of vascular implants and host arteries with 3D spatial information. However, the presence of streak artifacts caused by the coil mass may severely reduce the diagnostic quality of CBCT data, making this technique less valuable for imaging of patients with implants with high x-ray absorption.

We have shown that usage of MAR significantly improves image visibility by reducing the presence of metal streak artifacts caused by coil masses in VasoCT data acquired from patients after stent-assisted coil embolization. As a result, the overall visibility of surrounding vascular anatomy and neurovascular stents was improved with respect to data without MAR in $>90 \%$ of the cases. Streak artifacts that were extending beyond the actual coil mass in $56 \%$ without MAR were reduced to $4 \%$ with MAR. The observers agreed in $64 \%$ of the cases with MAR that the artifacts were not obscuring the host artery beyond the coil mass, which is lower than would be expected considering the drop from $56-4 \%$. This is potentially caused by the different interpretation of the subtle streak artifacts caused by the MAR algorithm as is shown in Figs $3 C$ and $4 B$. Prell et $\mathrm{al}^{30}$ have shown that $3 \mathrm{D}$ linear interpolation is less prone to introduce new artifacts than a technique that uses fewer dimensions. Factors that influence the outcome of the algorithm include the location and orientation of the coiled aneurysm with respect to the host artery and the size of the coil mass, which varied between cases. Overall, the visibility of VasoCT data was improved by MAR.

In principle, the MAR algorithm used in this study was based on a method previously proposed. ${ }^{30}$ In their work, Prell et al ${ }^{30}$ implemented an adaptive segmentation method to detect metallic objects in the primary reconstruction and correct for possible misalignment in the geometry calibration of the system. We found that the CBCT image quality of the primary reconstruction allowed segmentation by a fixed threshold value without causing oversegmentation or undersegmentation of the implants. In addition, the accuracy of the geometry calibration of the system used in our study was sufficient to perform forward projection without additional geometry corrections and image morphology. To limit the total processing time, a $1 \mathrm{D}$ linear interpolation routine was 
used to replace underexposed data in the raw projections. Although the results show that this simple interpolation method provides significant improvement in the overall visibility, the benefit of the use of advanced interpolation techniques should be assessed in further research. Furthermore, postprocessing methods such as attenuation-normalization and edge-enhancement as performed in a second correction step were not used here. ${ }^{29}$ The total reconstruction time with MAR was approximately $138 \mathrm{sec}-$ onds per dataset. Preliminary data show that prototype software with the use of the graphics processing unit reduced the total reconstruction time with MAR to approximately 50 seconds.

There are limitations to the use of MAR. Although successful removal of streak artifacts from CBCT results in a more appealing image in almost all cases, the diagnostic information may not always be improved compared with data without MAR. CBCT data generated during the second pass may contain some blurred regions and new subtle artifacts that are caused by the replacement of image content of the original x-ray data. However, these generally do not obscure the image content as severely as the artifacts caused by the coil mass. Furthermore, it should be noted that MAR is not capable of fully correcting all metal artifacts because data that are absent in the x-ray images as the result of photon starvation cannot be recovered. As a result of these limitations, diagnosis of recanalization at the aneurysm neck directly adjacent to the coil mass remains challenging. The development of more advanced methods is required to completely remove all artifacts caused by implants with high x-ray absorption, which may include alternative imaging protocols rather than postprocessing techniques.

\section{CONCLUSIONS}

We conclude that with the use of MAR on VasoCT data acquired for evaluation of patients who underwent stent-assisted coil embolization, overall reduced streak artifacts caused by coil masses, enhance the visibility of neurovascular stents and host arteries and therefore improve diagnostic quality.

\section{ACKNOWLEDGMENTS}

We gratefully acknowledge Philips Healthcare and NIH R01 CA128906 (PI: SV). The contents are solely the responsibility of the authors and do not represent the official views of the NIH or Philips Healthcare.

Disclosures: Daniel Ruijters—UNRELATED: Employment: Philips HealthCare. Peter van de Haar-UNRELATED: Employment: Philips Electronics, Netherlands, Comments: I am a full-time employee of Philips. Bart Carelsen—UNRELATED: Employment: Philips HealthCare, Comments: Clinical Scientist with the Business Innovation Unit Interventional X-ray. Srinivasan Vedantham-RELATED: Grant and Support for Travel to Meetings for the Study or Other Purposes: National Institutes of Health,* Comments: R01 CA128906; UNRELATED: Grants/Grants Pending: 1) National Institutes of Health, ${ }^{*}$ 2) Toshiba Medical Research Comments: 1) Multi-institution collaborative research involving Hologic, Inc; 2) Co-investigator in grant to Stephen J. Glick (Principal Investigator), University of Massachusetts Medical School. Matthew Gounis-UNRELATED: Consultancy: Stryker Neurovascular, Codman Neurovascular, Soteira, Surpass Medical, Comments: Fee-per-hour consultancy; Grants/Grants Pending: NIH, ${ }^{*}$ Codman Neurovascular, ${ }^{*}$ Stryker Neurovascular, ${ }^{*}$ eV3 Neurovascular,* Thrombolysis Science Inc,* Sanofi-Avantis,, Neuravi,* Neurointerventional Technologies Inc, ${ }^{*}$ Surpass Medical.* Ajay Wakhloo—RELATED: Grant: Philips Medical HealthCare, ${ }^{*}$ Comments: Research funding; UNRELATED: Board Membership: Surpass Medical; Consultancy: Surpass Medical, Boston Biomedical Associates, Stryker Neurovascular, Codman Neurovascular; Payment for Lectures (including ser- vice on speakers bureaus): Harvard Postgraduate Program, Miami Baptist Stroke Meeting; Stock/Stock Options: Surpass Medical (*Money paid to institution).

\section{REFERENCES}

1. Benitez RP, Silva MT, Klem J, et al. Endovascular occlusion of widenecked aneurysms with a new intracranial microstent (Neuroform) and detachable coils. Neurosurgery 2004;54:1359-67

2. Fiorella D, Albuquerque FC, Deshmukh VR, et al. Usefulness of the Neuroform stent for the treatment of cerebral aneurysms: results at initial (3-6-mo) follow-up. Neurosurgery 2005;56:1191-202

3. Higashida RT, Smith W, Gress D, et al. Intravascular stent and endovascular coil placement for a ruptured fusiform aneurysm of the basilar artery: case report and review of the literature. J Neurosurg 1997;87:944-49

4. Lanzino G, Wakhloo AK, Fessler RD, et al. Efficacy and current limitations of intravascular stents for intracranial internal carotid, vertebral, and basilar artery aneurysms. J Neurosurg 1999;91:538-46

5. Massoud TF, Turjman F, Ji C, et al. Endovascular treatment of fusiform aneurysms with stents and coils: technical feasibility in a swine model. AJNR Am J Neuroradiol 1995;16:1953-63

6. Wakhloo AK, Mandell J, Gounis MJ, et al. Stent-assisted reconstructive endovascular repair of cranial fusiform atherosclerotic and dissecting aneurysms: long-term clinical and angiographic followup. Stroke 2008;39:3288-96

7. Wakhloo AK, Schellhammer F, De Vries J, et al. Self-expanding and balloon-expandable stents in the treatment of carotid aneurysms: an experimental study in a canine model. AJNR Am J Neuroradiol 1994;15:493-502

8. Wakhloo AK, Linfante I, Silva CF, et al. Closed-cell stent for coil embolization of intracranial aneurysms: clinical and angiographic results. AJNR Am J Neuroradiol 2012;33:1651-56

9. Bendok BR, Parkinson RJ, Hage ZA, et al. The effect of vascular reconstruction device-assisted coiling on packing density, effective neck coverage, and angiographic outcome: an in vitro study. $\mathrm{Neu}$ rosurgery 2007;61:835-40

10. Piotin M, Blanc R, Spelle L, et al. Stent-assisted coiling of intracranial aneurysms: clinical and angiographic results in 216 consecutive aneurysms. Stroke 2010;41:110-15

11. Söderman $M$, Babic D, Holmin $S$, et al. Brain imaging with a flat detector c-arm. Neuroradiology 2008;50:863-68

12. Patel NV, Gounis MJ, Wakhloo AK, et al. Contrast-enhanced angiographic cone-beam CT of cerebrovascular stents: experimental optimization and clinical application. AJNR Am J Neuroradiol 2010;32:137-44

13. Snoeren RM, Söderman M, Kroon JN, et al. High-resolution 3D xray imaging of intracranial nitinol stents. Neuroradiology 2012;54:155-62

14. Glover GH, Pelc NJ. An algorithm for the reduction of metal clip artifacts in CT reconstructions. Med Phys 1981;8:799

15. Kalender WA, Hebel R, Ebersberger J. Reduction of CT artifacts caused by metallic implants. Radiology 1987;164:576-77

16. Abdoli M, De Jong JR, Pruim J, et al. Reduction of artefacts caused by hip implants in CT-based attenuation-corrected PET images using 2-D interpolation of a virtual sinogram on an irregular grid. Eur J Nucl Med Mol 2011;38:2257-68

17. Abdoli M, Ay MR, Ahmadian A, et al. Reduction of dental filling metallic artifacts in CT-based attenuation correction of PET data using weighted virtual sinograms optimized by a genetic algorithm. Med Phys 2010:6166-77

18. Veldkamp WJH, Joemai RMS, van der Molen AJ, et al. Development and validation of segmentation and interpolation techniques in sinograms for metal artifact suppression in CT. Med Phys 2010;37:620-28

19. Zhao S, Robeltson D, Wang G, et al. X-ray CT metal artifact reduction using wavelets: an application for imaging total hip prostheses. IEEE Trans Med Imaging 2000;19:1238-47

20. Lell MM, Meyer E, Kuefner MA, et al. Normalized metal artifact 
reduction in head and neck computed tomography. Invest Radiol 2012;47:415-21

21. Kachelrieß M, Watzke O, Kalender WA. Generalized multi-dimensional adaptive filtering for conventional and spiral single-slice, multi-slice, and cone-beam CT. Med Phys 2001;28:475-90

22. Bamberg F, Dierks A, Nikolaou K, et al. Metal artifact reduction by dual energy computed tomography using monoenergetic extrapolation. Eur Radiol 2011;21:1424-29

23. Wang G, Snyder DL, O'Sullivan JA, et al. Iterative deblurring for CT metal artifact reduction. IEEE Trans Med Imaging 1996;15:657-64

24. Robertson DD, Yuan J, Wang G, et al. Total hip prosthesis metalartifact suppression using iterative deblurring reconstruction. J Comput Assist Tomogr 1997;21:293-98

25. De Man B, Nuyts J, Dupont P, et al. An iterative maximum-likelihood polychromatic algorithm for CT. IEEE Trans Med Imaging 2001;20:999-1008

26. Hamill JJ, Brunken RC, Bybel B, et al. A knowledge-based method for reducing attenuation artefacts caused by cardiac appliances in myocardial PET/CT. Phys Med Biol 2006;51:2901-18

27. Kennedy JA, Israel $O$, Frenkel A, et al. The reduction of artifacts due to metal hip implants in CT-attenuation corrected PET images from hybrid PET/CT scanners. Med Biol Eng Comput 2007;45:553-62

28. Lemmens C, Faul D, Nuyts J. Suppression of metal artifacts in CT using a reconstruction procedure that combines map and projection completion. IEEE Trans Med Imaging 2009;28:250-60

29. Prell D, Kyriakou Y, Struffert T, et al. Metal artifact reduction for clipping and coiling in interventional C-arm CT. AJNR Am J Neuroradiol 2010;31:634-39

30. Prell D, Kalender W, Kyriakou Y. Development, implementation and evaluation of a dedicated metal artefact reduction method for interventional flat-detector CT. Br J Radiol 2010;83:1052-62

31. Feldkamp LA, Davis LC, Kress JW. Practical cone-beam algorithm. J Opt Soc Am 1984;1:612-19

32. Ruijters D, ter Haar Romeny BM, Suetens P. GPU-accelerated digitally reconstructed radiographs. BioMED'08: Proceedings of the Sixth IASTED International Conference on Biomedical Engineering. Anaheim, California: ACTA Press. 2008, pp. 431-435

33. Ruijters D, Homan R, Mielekamp P, et al. Validation of 3D multimodality roadmapping in interventional neuroradiology. Phys Med Biol 2011;56:5335-54

34. Fleiss JL, Cohen J. The equivalence of weighted kappa and the intraclass correlation coefficient as measures of reliability. Educ Psychol Meas 1973;33:613-19

35. Parkinson RJ, Eddleman CS, Batjer HH, et al. Giant intracranial aneurysms: endovascular challenges. Neurosurgery 2006;59: S103-12 